\title{
Sobre la función y objeto de la prueba
}

\section{Carlos Alberto Matheus López}

"Era evidente que la vida reserva siempre sorpresas mucho más complejas e imprevisibles y que el secreto consiste en dejarlas llegar sin interponerles castillos en el aire»

\section{Nociones previas}

Hemos de empezar este breve trabajo diferenciando necesariamente el trinomio conceptual relativo a la probática judicial ${ }^{1}$, constituido por los conceptos de medio de prueba, prueba y objeto de la prueba ${ }^{2}$. Con tal objetivo, podemos afirmar que la prueba constituye el procedimiento de probar o acreditar los hechos afirmados ${ }^{3}$. En tanto, el medio de prueba es el instrumento a través del cual se busca lograr la convic-

1 Término que preferimos utilizar, bajo la autoridad de Muñoz I. Sabaté, a fin de no crear confusión en el lector, respecto a la denominación y contenido de los siguientes tres conceptos a tratar.

2 Distinguen estos tres conceptos autores como Manuel Morón Palomino, Derecho Procesal Civil, Madrid, Marcial Pons, 1993, p. 269 y ss.; Prieto Castro y Leonardo Ferrandiz, Derecho Procesal Civil, $5^{\circ}$ edición, Madrid, Tecnos, 1989, p. 143 y ss.; Francisco Ramos Méndez, Enjuiciamiento Civil, Vol. I, J.M. Bosch Editor, Barcelona, 1997, p. 317 yss.

3 Comparten este criterio, autores como Prieto Castro, op. cit., p. 143; Jaime Guasp, Derecho Procesal Civil, Vol. I, Madrid, Instituto de Estudios Políticos, 1968, pp. 320 321; Andrés de la Oliva y Miguel Ángel Fernández, Derecho Procesal Civil, Vol. I, $4^{\circ}$ edición, Editorial Centro de Estudios Ramón Areces; Madrid, 1996, pp. 306 y ss.; Ramos, Op. cit., pp. 317-323. 
ción sobre el acaecimiento de un hecho particular ${ }^{4}$. Donde como obvia y necesaria consecuencia de lo antes dicho, debemos de indicar, en un primer término, que el objeto de la prueba viene constituido por los hechos afirmados por las partes ${ }^{5}$, teniendo que quedar ya sobrentendido desde ahora, que cuando nos referimos en este trabajo a hechos, hablamos en estricto de afirmaciones sobre hechos ${ }^{6}$, y esto por la sencilla razón que en realidad objeto de la prueba judicial no son directamente los hechos acaecidos en el pasado, dado que no se puede probar la verdad o falsedad de los hechos, los cuales pueden ser solamente constatados al momento de verificarse estos, y consecuentemente "pueden ser» o "no ser» y no «ser verdaderos» o «ser falsos». Y en tal sentido, queda claro que el objeto de la prueba son sólo afirmaciones, esto es, la "narración" que de los hechos acaecidos en el pasado hacen las partes frente al juez ${ }^{7}$.

\section{La función de la prueba}

Podemos señalar de modo pacífico que la prueba judicial desarrolla en el proceso una función que denominaremos «demostrativa», entendiéndose consecuentemente por ello que la función de la prueba, aquella dirigida a demostrar la verdad o falsedad de las afirmaciones factuales, debe ser asumida al interior del proceso mediante el recurso a un procedimiento de tipo racional. Y cuyos resultados -propiamente porque

4 Con tal parecer Prieto Castro, op. cit., p. 146. Entiende que medio de prueba es «el instrumento corporal o material cuya apreciación sensible constituye para el juez la fuente de donde ha de obtener los motivos para su convicción sobre la verdad (o no) del hecho que se trata de probar".

5 En ese sentido Morón, op. cit., p. 273. Nos señala que «las afirmaciones relativas a los hechos aducidos en el proceso pueden resultar necesitadas de demostración y por tanto integrar el objeto de la prueba»; del mismo modo De la Oliva, op.cit., p. 311. Entiende que «el objeto principal de la prueba son hechos. Mas exactamente podrá decirse que la actividad probatoria versa sobre afirmaciones de hechos o afirmaciones fácticas".

6 En tal sentido, si bien "comúnmente se afirma que la prueba recae sobre hechos. Esto, sin ser erróneo, es una expresión abreviada que hay que entender en su exacto significado. Nunca se prueban directamente hechos, lo que se prueba son siempre nuestras afirmaciones sobre diversos hechos" (Ramos, op. cit., p. 325).

7 Con tal parecer, Antonio Carrata, "Funzione dimostrativa della prova” en Rivista di Diritto Processuale Civile, Milano, Cedam, 2001, pp. 73-74. 
están fundados en un procedimiento de este tipo- son controlables desde el exterior por todos los destinatarios de la decisión, esto es, por el juez de la impugnación, y en general por todos los involucrados ${ }^{8}$.

Con idéntico parecer, considera Taruffo que «la prueba judicial desarrolla una función demostrativa, en cuanto provee un fundamento cognoscitivo y racional para la selección que el juez realiza individualizando una versión atendible y verídica de los hechos relevantes de la causa, y justificando racionalmente tal elección»?.

De tal forma, la reconstrucción de la prueba judicial en términos demostrativos importa asumir como fundamentos de esta, los siguientes $^{10}$ :

A. Que, al interior del proceso se puedan distinguir lógicamente los aspectos que pertenecen al juicio sobre los hechos, de aquellos atinentes al juicio de derecho.

B. Que, con relación a los aspectos que pertenecen al juicio sobre los hechos sea necesario organizar el procedimiento probatorio del juez, de acuerdo a una elaboración de tipo racional.

C. Que, sólo de tal modo es posible obtener el control de la selección llevada a cabo por el juzgador en la elaboración de su convencimiento sobre la veracidad o no de los enunciados factuales de la causa.

Quedándonos claro que los tres presupuestos se encuentran íntimamente vinculados entre sí, dado que su objetivo es dejar controlable desde el exterior el razonamiento probatorio del juzgador, lo cual impone el elaborar dicho razonamiento sobre bases racionales.

\subsection{Función demostrativa de la prueba y verdad}

En relación al tipo de resultado que se obtiene al interior del proceso como consecuencia de la actividad probatoria, no resulta de muy difícil observación que - por cuanto corresponde al resultado de la valoración de las pruebas por parte del juez- no se puede hablar en términos de verdad o certeza, ni aún menos sea oportuno continuar utilizando burdas ficciones como aquella de la distinción entre verdad

8 Carrata, op. cit., pp. 75-76.

9 Michele Taruffo, «Funzione de la prova: la funzione dimostrativa» en Rivista Trimestrale di Diritto e Procedura Civile», N³, Milano, Giuffrè-editore, 1997, p. 573; con similar criterio Francesco Carnelutti, La Prueba Civil, B. Aires, Ediciones Arayú, p. 39 y ss.

10 Carrata, op. cit., p. 76. 
material y verdad formal ${ }^{11}$, dado que hoy en día se encuentra pacíficamente admitido que el resultado de la valoración judicial es siempre la adquisición de la probabilidad de relación entre factum probandum y factum probans, y de una probabilidad no de tipo cuantitativa, por cuanto esta resulta dificilmente utilizable al interior del proceso, sino de tipo lógico ${ }^{12}$.

\subsection{Función demostrativa de la prueba y ordenamiento procesal}

Con relación a nuestro ordenamiento procesal civil, la función demostrativa de la prueba encuentra plena operatividad y no posee restricción alguna en su ejercicio, dado que esta se condice con un sistema de admisión de medios de prueba abierto ${ }^{13}$, y uno de valoración libre o apreciación conjunta ${ }^{14}$ de los medios de prueba, como son los que poseemos en nuestra normatividad procesal civil vigente. $Y$ en tal sentido, compartimos con Carrata que el mejor sistema probatorio será aquel que permita al juez experimentar todos los medios de prueba posibles y lícitos para obtener y determinar la verdad o falsedad del enunciado factual ${ }^{15}$.

Todavía aún, así nos encontrásemos en un ordenamiento procesal el cual estuviese regido por un sistema de admisión de medios de prueba cerrado, junto a un sistema de apreciación tasado de los medios de prueba, podremos afirmar que, si bien analizar la función demostrativa de la prueba en tal sistema procesal no es lo mismo que analizarla en el nuestro, no existe en realidad una diferencia desde el punto de

11 Distinción fundamentada en «la antítesis significativa entre la verdad material y la verdad formal o jurídica; el resultado de la búsqueda jurídicamente limitada o regulada no es, pues, la verdad material o, como diríamos mediante una eficaz redundancia, la verdad verdadera, sino una verdad convencional, que se denomina verdad formal, porque conduce a una indagación regulada en las formas, o verdad jurídica, porque se la busca mediante leyes jurídicas y no solo mediante leyes lógicas, y únicamente en virtud a esas leyes jurídicas remplaza a la verdad material» (Carnelutti, op. cit., pp. 20-21).

12 Con tal parecer Carrata, op. cit., p. 102.

13 Sistema reconocido en el texto del artículo $191^{\circ}$ del Código Procesal Civil vigente, el cual a la letra nos señala que "todos los medios de prueba, así como sus sucedáneos, aunque no estén tipificados en este código, son idóneos para lograr la finalidad prevista en el artículo $188^{\circ}$.

14 Como lo prescribe el tenor del artículo $197^{\circ}$ de nuestra actual norma procesal civil, al señalarnos que "todos los medios de prueba son valorados por el juez en forma conjunta, utilizando su apreciación razonada».

15 Carrata, op. cit., p. 82. 
vista epistemológico, en cuanto se debe reconocer que -en la lógica de la función demostrativa de la prueba- la prueba tiene (debe tener) siempre y como quiera que sea un fundamento racional sin importar el contexto en el cual es llamada a operar ${ }^{16}$.

\section{El objeto de la prueba y la necesaria distinción entre hechos y derecho}

Resulta interesante observar la distinción, que constituye el punto de partida de la teorética procesal sobre el objeto de la prueba, basada en la diferenciación fundamental entre los hechos y el derecho ${ }^{17}$, la cual si bien a primera vista nos parece cierta e irrefutable, olvida algo tan simple como el reconocer que el propio derecho, esto es su existencia en sí misma, no deja de ser un hecho en la realidad ${ }^{18}$, por lo cual la pretendida sólida distinción nos evidencia su artificialidad interna ${ }^{19}$.

16 Con tal parecer Carrata, op. cit., pp. 79-80.

Podemos observar así que «en el fondo de este principio existe, por una parte, la convicción que la actividad judicial, aún aquella de valoración de las pruebas deba será caracterizada por una serie mas o menos compleja de reglas, las cuales no representan otra cosa que, el decurso "histórico" de precisas tradiciones históricas, jurídicas, culturales, religiosas. Y por otro lado, la conciencia (del legislador in primis) que la determinación de la verdad o falsedad de los enunciados factuales al interior del proceso no puede ser perseguida "a toda costa" pero debe ser "constreñida" a soportar los límites que el mismo legislador ha considerado oportuno fijar, y esto porque -como observaba Carnelutti- la disciplina jurídica del procedimiento probatorio "viene constituida según el objeto (polírico) de obtener el conocimiento de los hechos controvertidos por la vía mas rápida y mas segura" (Carrata, op. cit., pp. 82-83).

17 Dado que «no cabe duda que en último término el complejo material de decisión reunido en un proceso es reducible, en cuanto a los elementos que lo componen, a uno de los grupos fundamentales apuntados: es decir, que en esencia está formado por hechos y normas de derecho objetivo" (Jaime Guasp, Juez y Hechos en el Proceso Civil, Barcelona, Bosch Casa Editorial, 1943, p. 12).

18 En este sentido Lluis Muñoz I Sabaté, Tratado de Probática Judicial, Vol. I, Barcelona, José María Bosch Editor, 1992, p. 30. Entiende que «el derecho es también un hecho, pero que para nosotros tiene la especial particularidad de que su conocimiento parece vital para el mantenimiento de la estructura social».

19 En tal forma, hemos de reconocer que «toda distinción entre hecho y derecho debe ser vista con mucha relatividad pues, los hechos que importan son los destinados a penetrar en el derecho. No se trata de que la distinción sea absoluta sino que para su 
Sin embargo, la utilidad de tal distinción consiste en saber que datos pueden y deben ser probados por las partes, y cuales han de ser aportados al proceso por el juez, estando exentos de prueba ${ }^{20}$, constituyendo la regla general que son objeto de prueba los hechos y no el derecho.

Podemos señalar sin embargo como excepciones a la regla general que el derecho no es objeto de prueba ${ }^{21}$, tanto a la costumbre como al derecho extranjero ${ }^{22}$, supuestos en los cuales su existencia y alcances

posible diferenciación es necesario eliminar toda visión antitética» (Sergi Guasch Fernández, El Hecho y el Derecho en la Casación Civil, Barcelona, José María Bosch Editor, 1998, p. 183).

20 Con tal parecer Regina Garcimartín Montero, El Objeto de la Prueba en el Proceso Civil, Barcelona, Cedecs Editorial, 1997, pp. 54-55; con similar opinión Giovanni Verde, "Prova» en Enciclopedia del Diritto, Vol. XXXVII, Varese, Giuffrè-editore, 1988, p. 588. Entiende que«el punto de partida es que sea posible al análisis distinguir el derecho (o como quiera que sea, el criterio de valoración) del hecho (o, como quiera que sea, el objeto de la valoración)».

21 En este sentido se pronuncia nuestra norma procesal civil al señalar en su artículo $190^{\circ}$ primer párrafo y adicionalmente en su inciso 4 que «los medios probatorios deben referirse a los hechos [...] Son también improcedentes los medios de prueba que tiendan a establecer $[\ldots]$ 4. El derecho nacional, que debe ser aplicado de oficio por los jueces».

De la lectura de esta disposición, debemos indicar que aquella resulta insuficiente al señalar tan solo el término «hechos", dado que debería indicar más técnicamente el término "hechos controvertidos". Sin embargo, de una interpretación sistemática del inciso 1 del mismo artículo $190^{\circ}$ (que seńala que no son objeto de prueba los hechos no controvertidos, pudiendo afirmarse por oposición que si lo son los hechos controvertidos), artículo $188^{\circ}$ (que vincula a los hechos y a los denominados puntos controvertidos, que no son otra cosa, por un lado, que hechos controvertidos), y $471^{\circ}$ del Código Procesal Civil (que determina la necesidad de fijar en la respectiva audiencia los puntos controvertidos, y aquí obviamente los hechos controvertidos), esta insuficiencia desaparece al quedar claro que son objeto de prueba los hechos controvertidos.

Por otra parte y a efectos comparativos, podemos observar que la vigente Ley de Enjuiciamiento Civil Española (Ley No 1/2000), señala en su artículo $281^{\circ}$ inciso 1, de manera más clara, que «la prueba tendrá como objeto los hechos que tengan relación con la tutela judicial que se pretenda obtener en el proceso».

22 En tal sentido Morón, op. cit., p. 276; de igual modo de la Oliva, op. cit., p. 315. Con igual criterio el artículo $190^{\circ}$ del Código Procesal Civil nos señala que "Los medios probatorios deben referirse [..] a la costumbre cuando ésta sustenta la pretensión [...] En el caso del derecho extranjero, la parte que lo invoque debe realizar actos destinados a acreditar la existencia de la norma extranjera y su sentidom. Del mismo modo, el Código Civil prescribe en su artículo $2052^{\circ}$ que slas partes litigantes pueden ofrecer las pruebas que tengan por conveniente sobre la existencia de la ley extranjera y su sentido. El juez puede rechazar o restringir los medios probatorios que no considere idóneos", señalan- 
deberán ser probados en el proceso. Por otra parte, y desde el extremo opuesto de esta misma regla, debemos señalar que las llamadas presunciones iuris et de iure constituyen en realidad una cuestión de derecho por lo que se encuentran también exentas de prueba ${ }^{23}$.

\section{El hecho como objeto de prueba}

A efectos de la claridad sistémica de nuestro trabajo hemos de precisar en primer término qué entendemos por hecho, y en ese sentido, diremos que aquel constituye todo suceso o acontecimiento que se ha producido en la realidad, el cual posee por ello existencia propia, sea ésta material o inmaterial ${ }^{24}$, así como una duración específica, pudiendo ser aquella determinada o determinable ${ }^{25}$.

do a continuación en su artículo $2053^{\circ}$ que «los jueces pueden de oficio o a pedido de parte, solicitar al Poder Ejecutivo que, por vía diplomática, obtenga de los tribunales del Estado cuya ley se trata de aplicar, un informe sobre la existencia de la ley y su sentidon. De modo similar, la Ley de Enjuiciamiento Civil Española (Ley No 1/2000), prescribe en su artículo $281^{\circ}$ inciso 2 que «también serán objeto la costumbre y el derecho extranjero. La prueba de la costumbre no será necesaria si las partes estuviesen conformes en su existencia y contenido y sus normas no afectasen al orden público. El derecho extranjero deberá ser probado en lo que respecta a su contenido y vigencia, pudiendo valerse el tribunal de cuantos medios de averiguación estime necesarios para su aplicación".

23 Con tal opinión De la Oliva, op.cit., p. 315; del mismo modo Garcimartín, op. cit., pp. 72-73. Entienden a este tipo de presunción como un supuesto de prueba prohibida por la ley; con similar parecer, entendemos que las mal llamadas presunciones iuris et de iure, constituyen en realidad una ficción legal, con lo cual pertenecen estrictamente al derecho y no a los hechos (Carlos Alberto Matheus López, «Análisis Crítico de la Presunción Legal Absoluta” en Libro Base del Primer Congreso Nacional de Derecho Procesal, Lima, Editorial Normas Legales, 1996, p. 126 y ss).

Reconoce este criterio el artículo $190^{\circ}$ inciso 3 de nuestra norma procesal civil al señalar que «Son también improcedentes los medios de prueba que tiendan a establecer [...] Los hechos que la ley presume sin admitir prueba en contrario".

24 Con tal opinión Prieto Castro, op. cit., p. 147.

Podemos señalar que constituyen hechos pertenecientes a la realidad inmaterial, por ejemplo, la intención, el conocimiento personal, la culpa, etc. Por otro lado, serán hechos correspondientes a la realidad inmaterial, la destrucción de un automóvil, la posesión de un inmueble, el deterioro de un bien mueble, etc.

25 Con tal parecer Garcimartín, op. cit., p. 51 y ss.

Resultarán hechos de duración determinable, aquellas actividades o situaciones que constituyan un estado o sucesión de hechos o actos susceptible de ser constatado, como por 
Hasta aquí y con tal concepto, en principio todos los hechos serían pasibles de constituir objeto de prueba, con lo cual se llegaría no sólo a un caos operativo, sino adicionalmente a la imposibilidad práctica de llevar a cabo en muchos casos un efectivo proceso ${ }^{26}$. Es por esta razón, que resulta adecuado precisar cuales son los hechos que constituyen estrictamente el objeto de la prueba, y en tal sentido, podemos afirmar que este último viene conformado por los hechos controvertidos, esto es, por aquellos hechos sobre los cuales las partes no se encuentran de acuerdo ${ }^{27}$. Resultando ello consecuencia del principio de contradicción por el que se rige el proceso civil, razón por la cual todo aquello que sea contradicho por alguna de las partes esta necesitado de prueba, resultando este tipo de hechos aquellos que constituyen el thema proband $2^{28}$.

Donde, tal afirmación nos obliga a diferenciar a partir de un criterio de exclusión cuáles son los hechos que no constituyen objeto de prueba, pudiendo afirmarse ante ello que estos importan a los hechos admitidos, los imposibles y los notorios.

\subsection{El hecho admitido}

Podemos entender por hecho admitido aquel alegado por una parte y cuya realidad es aceptada por la contraria, con lo cual no sólo deja de

ejemplo, la determinación del uso público o privado de un inmucble, la existencia o inexistencia de un contrato, etc.

26 Pues, tan sólo imagine por un momento estimado lector, un proceso en el cual deban de acreditarse todos los hechos afirmados por las partes.

27 En este sentido Garcimartín, op. cit., p. 51 y ss; con igual parecer pero con terminología distinta Juan Montero Aroca, Introducción al Derecho Jurisdiccional Peruano, Lima, Enmarce, 1999, p. 238. Entiende como "tema de prueba" a los hechos controvertidos.

Podemos observar las diversas posibilidades que surgen ante las recíprocas afirmaciones de una parte frente a otra. Así, ante la afirmación por parte de $A$ del hecho $x$, puede $B$ afirmar el hecho $-x$, con lo cual $x$ se convierte en un hecho controvertido. Por otro lado, ante la afirmación por $A$ del hecho $x$, puede $B$ afirmar que si $x$, constituyéndose así tal hecho como admitido. Asimismo, es posible que $A$ afirme el hecho $x$ y que $B$ no se pronuncie sobre aquel, con lo cual quedaría como un hecho sujeto a silencio, o bien que $B$ responda evasivamente, con lo cual quedaría como un hecho sujeto a respuesta evasiva. Finalmente, podría también suceder que ante los hechos afirmados por $A, B$ los niegue genéricamente (Con formulas tales como: «Niego y contradigo la demanda en todos sus extremos", "Absolutamente todo lo afirmado es falso", etc).

28 Con tal parecer, Ramos, Op. cit., p. 328. 
constituir objeto de prueba sino que se incorpora como premisa obligada de la sentencia a dictarse ${ }^{29}$. Pudiendo observarse que es el principio dispositivo el que en su aplicación veda la práctica de prueba sobre estos hechos, dado que en el proceso civil regido por este principio, las partes deben disponer de sus pretensiones y de los fundamentos de ellas, tanto jurídicas como fácticas ${ }^{30}$. Y en virtud de este principio, dichos hechos quedan fijados y de ellos ha de partir el Juez para dictar la sentencia, no pudiendo modificarlos de modo alguno ${ }^{31}$.

Quedándonos claro así que el hecho admitido es el resultado de la afirmación de un hecho por una parte frente a la otra, la cual manifiesta su conformidad con aquel, aceptación que podrá ser expresa o implícita, produciéndose la primera cuando una parte reconoce efectivamente el hecho aducido por la contraria, y la segunda cuando la actividad defensiva adoptada por una parte frente a la que alega el hecho presupone su aceptación ${ }^{32}$.

29 Con igual criterio Morón, op. cit., p. 273; del mismo modo Carnelutti, op.cit, p. 8. Entiende que "la afirmación de un hecho ya afirmado por la contraparte se llama admisión».

En este sentido, el artículo $190^{\circ}$ inciso 2 del Código Procesal Civil prescribe que «son también improcedentes los medios de prueba que tiendan a establecer [...]. Hechos afirmados por una de las partes y admitidos por la otra en la contestación de la demanda, de la reconvención en la audiencia de fijación de puntos controvertidos. Sin embargo, el Juez puede ordenar la actuación de medios probatorios cuando se trate de derechos indisponibles o presuma dolo o fraude procesales».

Debemos señalar, sin embargo, que la regulación de la norma resulta insuficiente dado que cabe la posibilidad que se den hechos admitidos también con la absolución del traslado de la contestación de la demanda o de la reconvención, en relación a nuevos hechos afirmados por una parte en su respectiva contestación, y la admisión de aquellos por la otra al momento de absolver el traslado respectivo.

Por otro lado, de forma parcialmente similar, la Ley de Enjuiciamiento Civil Española (Ley $N^{\circ} 1 / 2000$ ), prescribe en su artículo $281^{\circ}$ inciso 3 que «están exentos de prueba los hechos sobre los que exista plena conformidad de las partes, salvo en los casos en que la materia objeto del proceso éste fuera del poder de disposición de los litigantes".

30 De la Oliva, op. cit., pp. 312-313; con igual sentido Carnelutti, op. cit., pp. 9-10.

31 Con tal parecer, Ramos, Op. cit., pp. 327-328.

32 Morón, op. cit., p. 274; del mismo modo De la Oliva, op. cit., p. 306.

Cabe señalar como ejemplo de admisión expresa el reconocimien to por parte de $B$ de la afirmación de $A$ en relación al estado de conservación de un inmueble. En tanto será un caso de admisión implícita aquel en que $B$ excepciona la prescripción extintiva frente a la afirmación de $A$ respecto a la existencia de una obligación (con lo cual $B$ está aceptando que aquella existió). 
En relación al silencio, las respuestas evasivas y la negativa genérica, estas podrán estimarse como admisión de los hechos a que se refieren. Tratándose en estricto de una admisión presunta que no necesariamente exime a todos los hechos de prueba, pues su estimación dependerá del criterio judicial ${ }^{33}$.

\subsection{El hecho imposible}

Hemos de señalar en primer término que la posibilidad alude a la realidad de un hecho, esto es, a su capacidad de acontecer o no en el mundo real efectivamente. Debiéndose adicionalmente distinguir, como antes ya lo hicimos de manera general, el objeto de prueba y el medio de prueba imposibles respectivamente. En este sentido, la imposibilidad del medio de prueba alude a su impracticabilidad, o lo que es lo mismo, el medio de prueba no se podrá llegar a practicar o actuar en la realidad, como por ejemplo, en el caso de la declaración de testigo de un muerto. Por su parte la imposibilidad del objeto de prueba alude al hecho afirmado, el cual posee una clara incapacidad de producción real pero si es susceptible de practicarse prueba sobre él, sin obviamente arribar a un resultado útil, pues de lograrse este será inaceptable, como sucedería en el caso de preguntarse en una declaración de parte, sí el declarante estaba en el mismo momento en dos lugares distintos. Pese a ello, podemos observar que el efecto procesal que producen ambos supuestos es el mismo, esto es, el rechazo de la prueba por su inutilidad ${ }^{34}$.

Por otra parte, dentro de la imposibilidad de un hecho debemos distinguir dos tipos distintos de aquella, una que llamaremos física y

33 En este sentido Morón, op. cit., p. 274.

Con igual criterio el artículo $442^{\circ}$ inciso 2 del Código Procesal Civil nos señala que ael silencio, la respuesta evasiva o la negativa genérica pueden ser apreciadas por el juez como reconocimiento de verdad de los hechos alegados".

En tal sentido, podrá el hecho seguir dos caminos distintos, o ser considerado como admitido, con lo cual ya no será objeto de prueba, o no ser considerado como tal, con lo cual será entendido como hecho controvertido, y por ende constituirá objeto de prueba.

34 Garcimartín, op. cit., p. 65.

En este sentido el artículo $190^{\circ}$ inciso 1 de nuestra norma procesal civil nos señala que "son también improcedentes los medios de prueba que tiendan a establecer [...] hechos [...] imposibles». 
otra que denominaremos metafísica ${ }^{35}$. La primera importa la carencia absoluta de posibilidad que acontezca el hecho, como por ejemplo, que un padre sea mas joven que su hijo, y ello con total independencia del tiempo o lugar en que nos encontremos, del desarrollo científico u algún otro factor. En la segunda, por otra parte, tenemos un concepto relativo cuya determinación dependerá del lugar y tiempo en que nos hallemos, el avance científico u algún otro factor, con lo cual no se excluye totalmente la posibilidad de suceder de tal hecho, importando en realidad la dificultad de su efectiva producción. En tal sentido, si analizamos los ejemplos siguientes: el hecho que un trozo de hielo no se derrita con el calor del sol o que las personas cuando están nerviosas tartamudean. Podemos observar que existen diversos grados de dificultad del hecho, resultando más fácil de producirse el segundo hecho descrito que el primero.

Decíamos pues que la determinación de la imposibilidad física del hecho dependía de diferentes factores tales como el lugar de su producción. En este sentido, en relación al lugar, podemos señalar, por ejemplo, que es imposible que en nuestro país un día, es decir el lapso entre que sale el sol y se oculta, dure mas de veinticuatro horas, sin embargo en los polos tal lapso dura doce meses. Del mismo modo, es imposible que en Lima uno salga a una hora determinada y retorne luego a la misma hora o antes, cosa la cual si puede suceder al atravesarse en determinado instante el meridiano de Greenwich.

Por su parte en relación al tiempo, podemos indicar, por ejemplo, que un hecho que resultaba imposible en un momento determinado como la reducción física del territorio ártico, con el transcurso del tiempo se tornó en posible como producto de los desprendimientos y deshielos producidos por el efecto del agujero en la capa de ozono. Del mismo modo si bien resultaba imposible afirmar en una época la existencia de una laguna en cierto lugar, con el transcurso de los años y consecuente desvío de un río o afloramiento de corrientes de agua subterránea o fenómenos conocidos para nosotros como el de la corriente del niño, se torna en posible tal hecho.

Por otro lado, en relación al desarrollo científico podemos señalar como ejemplos que, hace algunas décadas era imposible determinar 
con un porcentaje del $99 \%$ de certeza, la vinculación genética entre dos seres, cosa la cual hoy en día es posible a través de la prueba del ADN. Tampoco resultaba posible varios años antes, representar gráficamente los olores $\mathrm{u}$ aromas, sin embargo hoy es plenamente posible a través de los cromatogramas. De igual modo, en la década del cincuenta era imposible que un hombre llegue físicamente a la luna, cosa la cual hoy es posible dada la existencia de naves espaciales.

De todo lo expuesto, podemos concluir que en el caso de la imposibilidad metafísica del hecho, la prueba siempre ha de ser rechazada por el juez, sin tomarla de modo alguno en cuenta. Pero, para el supuesto de imposibilidad física, el juzgador habrá de evaluar los factores que concurren en el caso concreto, admitiendo o denegando la prueba ${ }^{36}$.

\subsection{El hecho notorio}

La exigencia de economía procesal, se opone a la producción de pruebas innecesarias cuando la realidad de un hecho se encuentra incorporada al patrimonio cultural de un determinado momento y de una determinada circunstancia social, como es el caso de determinadas verdades históricas, geográficas, acontecimientos sociales locales o nacionales de especial relevancia etc, supuestos en los cuales nos encontramos ante un hecho notorio ${ }^{37}$. En ese sentido, Prieto Castro entiende como hecho notorio a aquellos que "por pertenecer a la ciencia, a la vida diaria, a la historia o al comercio social son conocidos o tenidos como ciertos por un circulo mas o menos grande de personas de cultura media» ${ }^{38}$.

37 Morón, op. cit., pp. 274-275.

Con tal parecer el artículo $190^{\circ}$ inciso 1 de nuestra norma procesal civil señala a la letra que «Son también improcedentes los medios de prueba que tiendan a establecer [...] hechos [...] notorios o de pública evidencia».

Resulta necesario señalar que esta disposición utiliza innecesariamente el término "pública evidencia", el cual se encuentra ya comprendido dentro del concepto de "hecho notorio", razón por la cual bastaba tan sólo referirse a este último.

Por otro lado, con similar criterio, la Ley de Enjuiciamiento Civil Española (Ley No $1 /$ 2000 ), prescribe en su artículo $281^{\circ}$ inciso 4 que «no será necesario probar los hechos que gozan de notoriedad absoluta y general".

38 Prieto Castro, op. cit., p. 148. Denominando el autor a estos hechos indistintamente como "notorios o de fama pública". 
En tal forma, la notoriedad de un hecho importa un grado especial y más intenso del conocimiento que provoca en el que esta destinado a recibirlo. Pues perteneciendo el dato a la cultura o experiencia común de los hombres, su conocimiento positivo, una vez que se logra, reviste extraordinaria garantía. No siendo así notorios aquellos hechos que el órgano jurisdiccional pueda conocer oficial o privadamente sino aquellos que disfrutan de un reconocimiento general en el lugar y tiempo en que el proceso se desarrolla ${ }^{39}$. Se distingue también por su expansión o magnitud una notoriedad general y una local ${ }^{40}$, aunque tal distinción obviamente no pueda determinarse en la realidad de manera absoluta.

Ahora bien, como para el caso del hecho imposible, el hecho notorio se encuentra también limitado por el lugar y tiempo, pero a diferencia del primero en que estos criterios se determinan al momento de su acontecimiento, en el segundo aquellos se analizan al momento en que se da el proceso, no siendo relevante aquí la producción efectiva o no del hecho, sino el conocimiento generalizado de aquel, el cual lo exime de prueba ${ }^{41}$.

En este sentido, la notoriedad no es una cualidad de un hecho que le convenga siempre, en todo lugar y de una vez por todas, puesto que aquella varía según el tiempo y el espacio. Lo que es notorio en Lima puede no serlo en Amazonas, y lo que hoy sabemos todos (o estamos en aptitud de conocer) dentro de un año o meses lo habremos olvidado ${ }^{42}$.

Debemos precisar además que la notoriedad del hecho puede alcanzarse tanto por medio de un conocimiento directo de aquel o bien a través de un conocimiento indirecto de este por haber sido difundido a través de medios de comunicación, o haber sido transmitido por medio oral ${ }^{43}$.

39 Guasp, Derecho..., op. cit., p. 330 . Incluyendo el autor dentro de los hechos notorios a aquellos que denomina "evidentes o axiomáticos».

40 Con tal parecer Prieto Castro, op. cit., p. 148; con similar parecer Giuseppe De Stefano, "Fatto Notorio" en Enciclopedia del Diritto, VI. XVI, Varese, Giuffrè-editore, 1967, p. 1006. Entiende que «no está excluido que el juez pueda utilizar aquella fuente de conocimiento la así denominada notoriedad local".

Podemos señalar como ejemplos de notoriedad local el hecho que un determinado alcalde sea conocido tan sólo en su distrito o provincia. Mientras que será un supuesto de notoriedad general el hecho que todo el país conozca quien es el presidente de la república.

41 En este sentido Garcimartín, op. cit., p. 76.

42 Con tal parecer De la Oliva, op. cit., p. 313.

43 Con similar criterio Garcimartín, op. cit., p. 78. 
Podemos de todo lo expuesto señalar que se caracteriza el hecho notorio por su generalidad, esto es, el conocimiento difundido y amplio de este dentro del ámbito del cual se trate, o lo que es lo mismo, que se conozca o pueda ser conocido por un número indeterminado de personas ${ }^{44}$. En ese sentido, la notoriedad debe ser general, no importando por ello que el hecho sea notorio para el juez, sino para toda la comunidad, de la cual él es parte o no, razón por la cual no podemos aceptar la así llamada notoriedad judicial ${ }^{45}$, dado que la notoriedad supone que un hecho sea conocido por un mayor o menor número de personas y entre ellas por el juez ${ }^{46}$.

Será un hecho notorio por conocimiento directo, por ejemplo, un gran incendio producido en un determinado pueblo y observado por la mayoría de sus habitantes. En tanto resultará un hecho notorio por conocimiento indirecto, por ejemplo, la destrucción de una determinada autopista la cual ha sido ampliamente difundida por medios televisivos.

44 En tal sentido Garcimartín, op. cit., p. 77; del mismo modo De Stefano, op. cit., p. 1003. Entiende que «este requisito se impone obviamente porque el reducir las características del notorio a la sola certeza individual del órgano jurisdiccional colisiona contra los principios que prohíben reunir la calidad de juez y de testigo y de utilizar para la decisión, en cuanto a los hechos singulares, el saber privadon; del mismo modo Verde, op. cit., p. 619. Entiende que "por ser conocidos por la comunidad de la cual el juez es componente, excluye que este, al utilizarlos, asuma la posición de parte. Mas bien la pertenencia del hecho al patrimonio de la cultura común, si bien por un lado impide que el juez pueda allí llevar a cabo una utilización arbitraria e incontrolable, por otro lado es garantía que sobre este pueda darse confianza para fundar la decisión judicial".

45 De aceptar que el hecho sea solo notorio para el juez, y no para la generalidad de sujetos, aquello importaría que ante un cambio de juzgador en un proceso, el cual no sea oriundo del lugar donde se producen los hechos, entonces aquellos no serían de manera alguna notorios frente a él. Del mismo modo, el colegiado que en apelación conozca de un determinado proceso, y a su vez se ubique en lugar distinto al de los hechos, entonces tampoco debería aceptar como notorios tales hechos frente a él. Consideramos por todo ello que la notoriedad del hecho no es respectiva al juez, sino a la generalidad de individuos del ámbito del cual se trate, del cual el juzgador podrá formar parte o no, como en los dos casos antes vistos, caso en el cual deberá procurarse por sus propios medios el conocimiento de tales hechos; en contra Prieto Castro, op. cit., p. 148; del mismo modo De Stefano, op. cit., p. 101. Comprende existente a "la así denominada notoriedad judicial, entendida como conocimiento que el juez tiene de ciertos hechos por razón de su oficio. Se considera que tales hechos pueden ser utilizados por el juez sin prueba ni alegación de parte y no es dudoso que tal reconocimiento responda a motivos de buen sentido, que sería absurdo negar».

46 Con idéntica opinión, Ramos, Op. cit., p. 328. 
Donde al requisito de la generalidad debe añadirse aquel de la certeza ${ }^{47}$, la cual deviene en necesaria a fin que se produzca el efecto procesal de la no necesidad de prueba, siendo indiferente que aquella sea positiva o negativa, esto es, que el hecho sea notoriamente falso o notoriamente verdadero ${ }^{48}$.

Por otra parte debemos señalar que el hecho notorio debe ser afirmado por las partes como cualquier otro hecho, no pudiendo ser traído al proceso por mera decisión del juzgador ${ }^{49}$.

Finalmente, debemos indicar que la regla notoria non egent probatione hasta ahora desarrollada, posee una excepción y esta viene dada para los supuestos en que sea la ley misma la que exija la prueba de la notoriedad ${ }^{50}$.

\section{Consideraciones finales}

Cabe señalar que en el ordenamiento procesal peruano, conforme lo prevé el último párrafo del artículo $190^{\circ}$ del Código Procesal Civil, la declaración de improcedencia de los medios de prueba destinados a establecer los hechos antes descritos, se lleva a cabo por el juzgador en la audiencia de fijación de puntos controvertidos del procedimiento respectivo del cual se trate. En tal sentido, el juzgador admitirá todos los medios de prueba relativos a hechos controvertidos, y del mismo modo, podrá de acuerdo a su particular criterio establecer cuáles constituyen en el proceso hechos imposibles, notorios o admitidos.

47 De Stefano, op. cit., p. 1003. Entiende que al «requisito de la generalidad, que parece así consustancial al de conocimiento notorio, debe por esto añadirse aquel de certeza».

48 Garcimartín, op. cit., p. 78.

Será un hecho notoriamente falso que los objetos no caen al suelo al ser atraídos por la gravedad. En tan to será un hecho notoriamente verdadero que la tierra gira alrededor del sol.

49 Con tal parecer Garcimartín, op. cit., p. 86; con criterio parcialmente similar De Stefano, op. cit., p. 1009. Entiende que "las afirmaciones de parte permanecen indispensables para los hechos fundamentales que constituyen las razones de la demanda, ósea la causa petendi», en contra Guasp, Juez..., op.cit., pp. 20-21

50 En nuestro ordenamiento aquello sucede por ejemplo en lo dispuesto por los artículos $186^{\circ}$ a $188^{\circ}$ del Decreto Legislativo No 823 «Ley de Propiedad Industrial», caso en el cual debe probarse la existencia de la notoriedad de la marca para poder bencficiarse con sus alcances. 
Y de determinar la existencia de alguno de ellos, declarará improcedentes los medios de prueba relativos a estos, como obvia consecuencia de todo lo anteriormente señalado. Por otro lado, de no encontrarse conformes las partes con tal decisión, por no considerar que un determinado hecho sea imposible, admitido o que resulte notorio, podrán apelar de la decisión sin efecto suspensivo. Donde de ser revocada la resolución por el superior antes que el juez dicte sentencia, este último deberá actuar el medio de prueba respectivo. Caso contrario será el superior el que actuará el medio de prueba antes de sentenciar. 